\title{
Clinical Application of Three-Dimensional Printing Technology in Craniofacial Plastic Surgery
}

\author{
Jong Woo Choi ${ }^{1}$, Namkug Kim ${ }^{2}$ \\ Departments of ${ }^{1}$ Plastic Surgery and ${ }^{2}$ Convergence Medicine, Asan Medical Center, Ulsan University College of Medicine, Seoul, Korea
}

Three-dimensional (3D) printing has been particularly widely adopted in medical fields. Application of the 3D printing technique has even been extended to bio-cell printing for 3D tissue/ organ development, the creation of scaffolds for tissue engineering, and actual clinical application for various medical parts. Of various medical fields, craniofacial plastic surgery is one of areas that pioneered the use of the 3D printing concept. Rapid prototype technology was introduced in the 1990s to medicine via computer-aided design, computer-aided manufacturing. To investigate the current status of 3D printing technology and its clinical application, a systematic review of the literature was conducted. In addition, the benefits and possibilities of the clinical application of 3D printing in craniofacial surgery are reviewed, based on personal experiences with more than 500 craniofacial cases conducted using 3D printing tactile prototype models.

Keywords Three-dimensional printing / Computer simulation / Craniofacial Abnormalities / Orthognathic surgery / Surgical flap
Correspondence: Jong Woo Choi Department of Plastic Surgery, Asan Medical Center, Ulsan University College of Medicine, 88 Olympic-ro 43-gil, Songpa-gu, Seoul 138-736, Korea

Tel: +82-2-3010-3604

Fax: +82-2-476-7471

E-mail: pschoi@amc.seoul.kr

No potential conflict of interest relevant to this article was reported.

Received: 24 Feb 2015 • Revised: 2 Mar 2015• Accepted: 3 Mar 2015

pISSN: 2234-6163 • elSSN: 2234-6171 • http://dx.doi.org/10.5999/aps.2015.42.3.267• Arch Plast Surg 2015;42:267-277

\section{INTRODUCTION}

Three-dimensional (3D) printing applications have generated increased interest in recent years. Many countries are trying to develop a 3D printing industry and apply this technique to various fields, including business, fashion, the mechanical engineering and medicine $[1,2] .3 \mathrm{D}$ printing has been particularly widely adopted in medical fields. Although 3D printing with tactile rapid prototype (RP) models was developed in the 1980s, this technique is being revisited due to its value and efficacy [3-5]. Application of the $3 \mathrm{D}$ printing technique has even been extended to bio-cell printing for 3D tissue/organ development, the creation of scaffolds for tissue engineering, and actual clinical application for various medical parts $[6-8]$.
$3 \mathrm{D}$ printing technology is not a new concept and has already been used in the mock formation of various products, including cellular phones [2]. However, as this technology has evolved, the extent of the application has greatly expanded, especially in medicine. Its increased use is largely because it can provide an individual product in a short period of time, which suits the goal of individualized medicine where each patient requires a specific, tailored, therapeutic approach. In contrast, most industrial products are mass produced and have the same dimensions and size. As one would expect, in medicine, patients have different anatomies and needs. Because one of the aims of contemporary medicine is to implement personalized medicine, the $3 \mathrm{D}$ printing technique should be applied to support such approaches as it can provide a patient-specific product in a short period of time 
without sacrificing costs or benefits.

Of various medical fields, craniofacial plastic surgery is one of areas that pioneered the use of the $3 \mathrm{D}$ printing concept. $\mathrm{RP}$ technology was introduced in the 1990s to medicine via computeraided design, computer-aided manufacturing (CAD-CAM). The medical models or bio-models based on the $3 \mathrm{D}$ printing technique represent 1:1 scale portions of the human anatomical region of interest obtained via 3D medical imaging [3]. The procedure for the fabrication of medical models consists of multiple steps: (1) acquisition of high-quality volumetric 3D image data of the anatomical structure to be modeled, (2) 3D image processing to extract the region of interest from the surrounding tissues, (3) mathematical surface modeling of the anatomic surfaces, (4) formatting of data for rapid prototyping, (5) model building, and (6) quality assurance of the model and its dimensional accuracy [9].

For example, because patients requiring craniofacial surgery tend to have very specific malformations or deformities, mostly in the bone, a 3D printing prototype model can greatly assist with preoperative evaluation and intraoperative procedures. Medical modeling in craniofacial surgery based on 3D printing has mainly been developed over the last 15 years and can incorporate: (1) aiding in the production of surgical implants, (2) improving surgical planning, (3) acting as an orientation aid during surgery, (4) enhancing diagnostic quality, (5) assisting preoperative simulation, (6) achieving a patient's consent prior to surgery, and (7) preparing a template for resection for surgeons (Winder and Bibb [3]), as well as providing an educational tool for medical students and residents [10]. The present article reviews the current state of 3D printing technology, its clinical application in plastic surgery, and the future direction of its development.

\section{METHODS}

To investigate the current status of 3D printing technology and its clinical application, a systematic review of the literature was conducted using PubMed. A PubMed search was performed based on title-abstract sifting by one author. Our inclusion criteria involved the following keywords: "3D printing", "craniofacial”, "rapid prototype", and "medical modeling”. After title-abstract sifting, 55 articles were retained; 20 articles were excluded after being read. Thus, 35 articles were selected for review. In addition, the benefits and possibilities of the clinical application of $3 \mathrm{D}$ printing in craniofacial surgery are reviewed, based on personal experiences with more than 800 craniofacial cases conducted using $3 \mathrm{D}$ printing tactile prototype models.

\section{RESULTS}

\section{Review of current $3 \mathrm{D}$ printing techniques used in craniofacial surgery $[11]$}

3D printing technology can be classified by the techniques, the materials or aimed deposition process: The technical type classification includes Stereolithography (SL), the selective laser sintering (SLS), 3D printing (Binder-Jet), fused deposition modeling (FDM), direct metal laser sintering (DMLS), laminated object manufacturing (LOM), and electron beam melting (EBM); The material classification includes thermoplastic, metal powder, ceramic powder, eutectic metals, alloy metal, photopolymer, paper, foil, plastic film, titanium alloys, etc; The aimed deposition process classification includes polyjet modeling based on drop-on-drop deposition, and fused deposition modeling based on continuous deposition. The most frequently used representative methods will be reviewed (Table 1). For medical usage, after the computed tomography (CT) scan, the rendering of the DICOM data and transformation into stereolithography data files takes a maximum of 30 minutes. In general, $3 \mathrm{D}$ printer accuracy would depend on the accuracy of CT scans, especially of which thickness should be as thin as possible ( 1 to $2 \mathrm{~mm}$ is a good compromise for a skull study).

Liquid based 3D printing technology

Stereolithography (SL or SLA)

SL has been the most widely used 3D technique for craniofacial surgery, since it was first applied for grafting a skull defect in 1994 [12]. The SL RP system consists of a bath of photosensitive resin, a model-building platform, and an ultraviolet (UV) laser for curing the resin. A mirror, computer controlled, is used to guide the UV laser focus onto the surface of the resin and cure the resin on a slice-by slice basis. These slice data are fed into the RP machine that directs the exposure path of the UV laser onto the surface of the resin. The layers are cured sequentially and bind together to form a solid object, beginning from the bottom of the model and building upward. Each new layer of resin is wiped across the surface of the previous layer using a wiper blade before being exposed and cured. The model is then removed from the bath and cured for an additional period of time in a UV cabinet [11]. Generally, SL is considered to provide the greatest accuracy and best surface finish of any $\mathrm{RP}$ technology. The model material is robust, slightly brittle, and relatively light $[13,14]$. SL accuracy is $1.2 \mathrm{~mm}$ (range, $0-4.8 \mathrm{~mm}$ ) for skull base measures, $1.6 \mathrm{~mm}$ (range, $0-5.8 \mathrm{~mm}$ ) for midface measures, $1.9 \mathrm{~mm}$ (range, 0-7.9 mm) for maxilla measures, and $1.5 \mathrm{~mm}$ (range, $0-5.7 \mathrm{~mm}$ ) for orbital measures. The mean differences in defect dimensions are $1.9 \mathrm{~mm}$ (range, $0.1-5.7 \mathrm{~mm}$ ) 
Table 1. A comparison of current three-dimensional (3D) printing technologies

\begin{tabular}{|c|c|c|c|}
\hline 3D printing technology & Materials & Aimed deposition process & Mechanism \\
\hline \multicolumn{4}{|l|}{ Liquid base } \\
\hline Stereolithography (SL) & Photo polymer & & $\begin{array}{l}\text { Layer-by-layer curing method based on ultraviole } \\
\text { (UV) laser focusing on photosensitive polymer }\end{array}$ \\
\hline Polyjet or multijet printing & $\begin{array}{l}\text { Acrylonitrile butadiene styrene (ABS), } \\
\text { Acryl }\end{array}$ & Drop-on-drop deposition & $\begin{array}{l}\text { Printer head that extrude the liquid resin and wax } \\
\text { with UV laser }\end{array}$ \\
\hline \multicolumn{4}{|l|}{ Powder base } \\
\hline Selective laser sintering (SLS) & $\begin{array}{l}\text { Thermoplastic } \\
\text { Metals powder }\end{array}$ & & \\
\hline 3D printing (3DP) & Plastic powder & Drop-on-powder deposition & \\
\hline \multirow[t]{2}{*}{ Direct metal laser sintering (DMLS) } & Alloy metal & & \\
\hline & Ceramic powder & & \\
\hline Electron beam melting (EBM) & Titanium alloys & & \\
\hline \multicolumn{4}{|l|}{ Solid base } \\
\hline \multirow[t]{3}{*}{ Fused deposition modeling (FDM) } & Thermoplastic & Continuous deposition & \\
\hline & Eutectic metals & & \\
\hline & ABS & & \\
\hline \multirow[t]{3}{*}{ Laminated object manufacturing (LOM) } & Paper & & \\
\hline & Foil & & \\
\hline & Plastic film & & \\
\hline
\end{tabular}

for unilateral maxillectomy, $0.8 \mathrm{~mm}$ (range, $0.2-1.5 \mathrm{~mm}$ ) for bilateral maxillectomy, and $2.5 \mathrm{~mm}$ (range, models may be more prone to error than those of other craniofacial regions because of the presence of thin walls and small projections. Choi et al. [13] found that the absolute mean deviation between an original dry skull and an SL RP model over 16 linear measurements was $0.62 \pm 0.5 \mathrm{~mm}(0.56 \% \pm 0.39 \%)$ [15]. Schicho et al. [15] compared the accuracy of computed tomography (CT) and SL models. The accuracy for SL models expressed as the arithmetic mean of the relative deviations ranged from $0.8 \%$ to $5.4 \%$, with an overall mean deviation of $2.2 \%$. The mean deviations of the investigated anatomical structures ranged from $0.8 \mathrm{~mm}$ to 3.2 $\mathrm{mm}$. An overall mean of deviations (comprising all structures) of $2.5 \mathrm{~mm}$ was found.

\section{Polyjet modeling}

Polyjet modeling is performed by jetting state-of-the-art photopolymer materials in ultra-thin layers $(16 \mu \mathrm{m})$ onto a build tray layer by layer, until the model is completed. Each photopolymer layer is cured by UV light immediately after it is jetted, producing fully cured models that can be handled and used immediately without post-curing. The gel-like support material used, which is specially designed to maintain complicated geometries, is easily removed by hand and water jetting [11]. At present, this technique is too time consuming and, therefore, too expensive to be used in craniofacial surgery clinical applications. Ibrahim et al. reported a dimensional error of $2.14 \%$ in reproducing a dry mandible when using this technique [16].
Powder based 3D printing technology

Selective laser sintering (SLS)

The SLS technique uses a $\mathrm{CO}_{2}$ laser beam to selectively fabricate models in the following way. First, the 2D slice data are fed into the SLS machine that directs the exposure path of the laser over a thin layer of powder previously deposited on the build tray and leveled with a roller. The laser heats the powder particles, fusing them to form a solid layer, and then moves along the $\mathrm{X}$ and $\mathrm{Y}$ axes to design the structures according to the $\mathrm{CAD}$ data. After the first layer fuses, the build tray moves downward, and a new layer of powder is deposited and sintered and the process is repeated until the object is completed. The prototype surface is finished by sandblasting [11]. The SLS prototype is opaque, and its surface is abrasive and porous. Prototype fabrication time is 15 hours. The accuracy of the SLS model is relatively high, with maximum standard errors of 0.1 to $0.6 \mathrm{~mm}$. Because of the high cost of the materials, several parts are fabricated simultaneously. The long fabrication time for the SLS technique (16 hours) is close to the time required for fabrication with the SL system [17].

\section{D printing (BinderJet)}

The $3 \mathrm{D}$ printing system uses a print head to selectively disperse a binder onto powder layers. This technology has a lower cost than similar techniques. First, a thin layer of powder is spread over a tray using a roller similar to that used in the SLS system. The print head scans the powder tray and delivers a continuous jet of a solution that binds the powder particles as it touches them.

No support structures are required while the prototype is being 
fabricated, because the surrounding powder supports the unconnected parts. When the process is complete, the surrounding powder is aspirated. In the finishing process, the prototype surfaces are infiltrated with a cyanoacrylate-based material to harden the structure [17]. The printing technique enables the formation of complex geometrical structures, such as hanging partitions inside cavities, without artificial support structures [11]. The printing and infiltration process takes approximately 4-6 hours. The material costs for the construction of each model are 150-200 EUR [17]. The 3D printers used in this process are relatively inexpensive (25,000 GBP), have fast build times (4 hours for a full skull), and are easy to maintain. Additionally, 3D printers are cost effective $\left(1 \mathrm{GBP} / \mathrm{cm}^{3}\right)$, associated with low waste, accurate $( \pm 0.1$ $\mathrm{mm}$ in the $\mathrm{Z}$ plane, $\pm 0.2 \mathrm{~mm}$ in the $\mathrm{X}$ and $\mathrm{Y}$ planes), have small dimensions, and can make hard, soft, or flexible models. These printers can also be used to identify different types of body tissue depending on the predefined threshold setting selected. Silva et al. [17] reported a mean dimensional error of $2.67 \%$ in prototypes produced using $3 \mathrm{D}$ printing technologies in comparison with a dry human skull [17].

\section{Solid based 3D printing technology}

FDM uses a similar principle to SL in that it builds models on a layer-by-layer basis. The main difference is that the layers are deposited as a thermoplastic that is extruded from a fine nozzle. A commonly used material for this procedure is acrylonitrile butadiene styrene (ABS). The 3D model is constructed by extruding the heated thermoplastic material onto a foam surface along a path indicated by the model data. Once a layer has been deposited, the nozzle is raised by between 0.278 to $0.356 \mathrm{~mm}$ and the next layer is deposited on top of the previous layer. This process is repeated until the model is completed [11]. As with SL, support structures are required for FDM models, because time is needed for the thermoplastic to harden and the layers to bond together [18].

\section{Bench-to-bed process for 3D printing-based tactile models (Fig. 1)}

(1) CT scan: after the patient is scanned via CT, DICOM files should be exported. Less than a 1-mm CT slice thickness is recommended.

(2) Data conversion: DICOM data are imported and converted to STL files. Rendering of CT scan DICOM data into STL data

\section{Fig. 1. Process of 3D printing}

The overall process of three-dimensional (3D) printing in craniofacial surgery. After the patient is scanned via computed tomography (CT), DICOM files should be exported. Less than a 1-mm CT slice thickness is recommended. DICOM data are imported and converted to stereolithograhy (STL) files. Rendering of CT scan DICOM data into STL data files takes about 30 minutes. Converted 3D files are uploaded into the 3D printer. Rapid prototyping follows using layer-by-layer Stereolithographic accumulation. The rapid prototype model is then fabricated on plaster via jetting of the materials. The materials consist of plaster $(<90 \%)$, vinyl polymer $(<20 \%)$, and carbohydrate $(<10 \%)$. The printing and infiltration process takes about 4-6 hours. Finally, unsintered sections should be removed.

\section{Process of 3D printer}

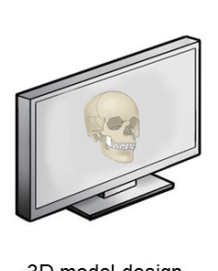

3D model design on the computer

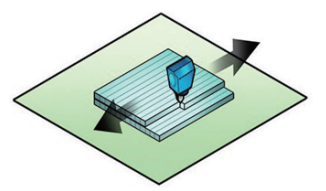

A print head to selectively disperse a binder onto powder layers
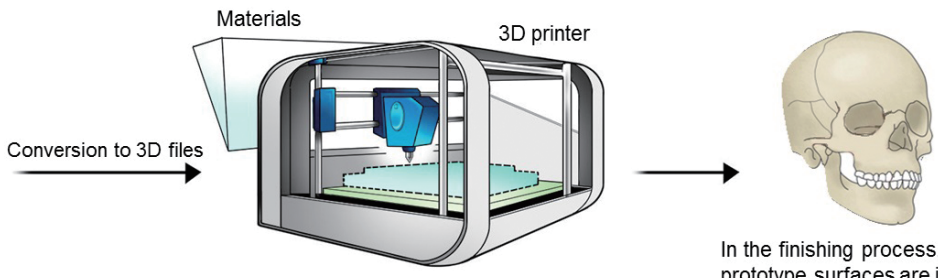

In the finishing process, the prototype surfaces are infiltrated with a cyanoacrylate-based material to harden the structure

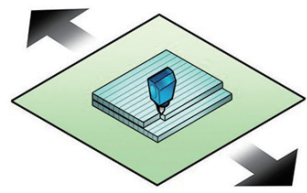

Platform moved according to the movement of nozzle

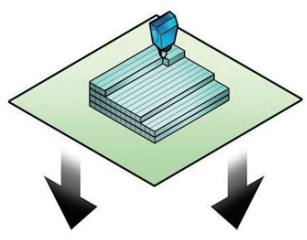

The print head scans the powder tray and delivers a continuous jet of a solution that binds the powder particles 
files takes about 30 minutes.

(3) Fabrication: converted 3D files are uploaded into the 3D printer. Rapid prototyping follows using layer-by-layer stereolithographic accumulation. The RP model is then fabricated on plaster via jetting of the materials. The materials consist of plas- ter $(<90 \%)$, vinyl polymer $(<20 \%)$, and carbohydrate $(<10 \%)$. The printing and infiltration process takes about 4-6 hours.

(4) Filtering: unsintered sections should be removed.

Fig. 2. Skull reconstruction with split calvarial bone grafting

Skull reconstruction with split calvarial bone grafting. (A) When the ideal donor site which has proper contour and thickness is determined, the three-dimensional (3D) printed tactile model can be very helpful for the split calvarial bone grafting. (B) Pre- and postoperative frontal views. (C) $3 \mathrm{D}$ printed titanium implant for the correction of the calvarial bone defect. (D) Preoperative and postoperative results. Temporal muscle atrophy is shown.
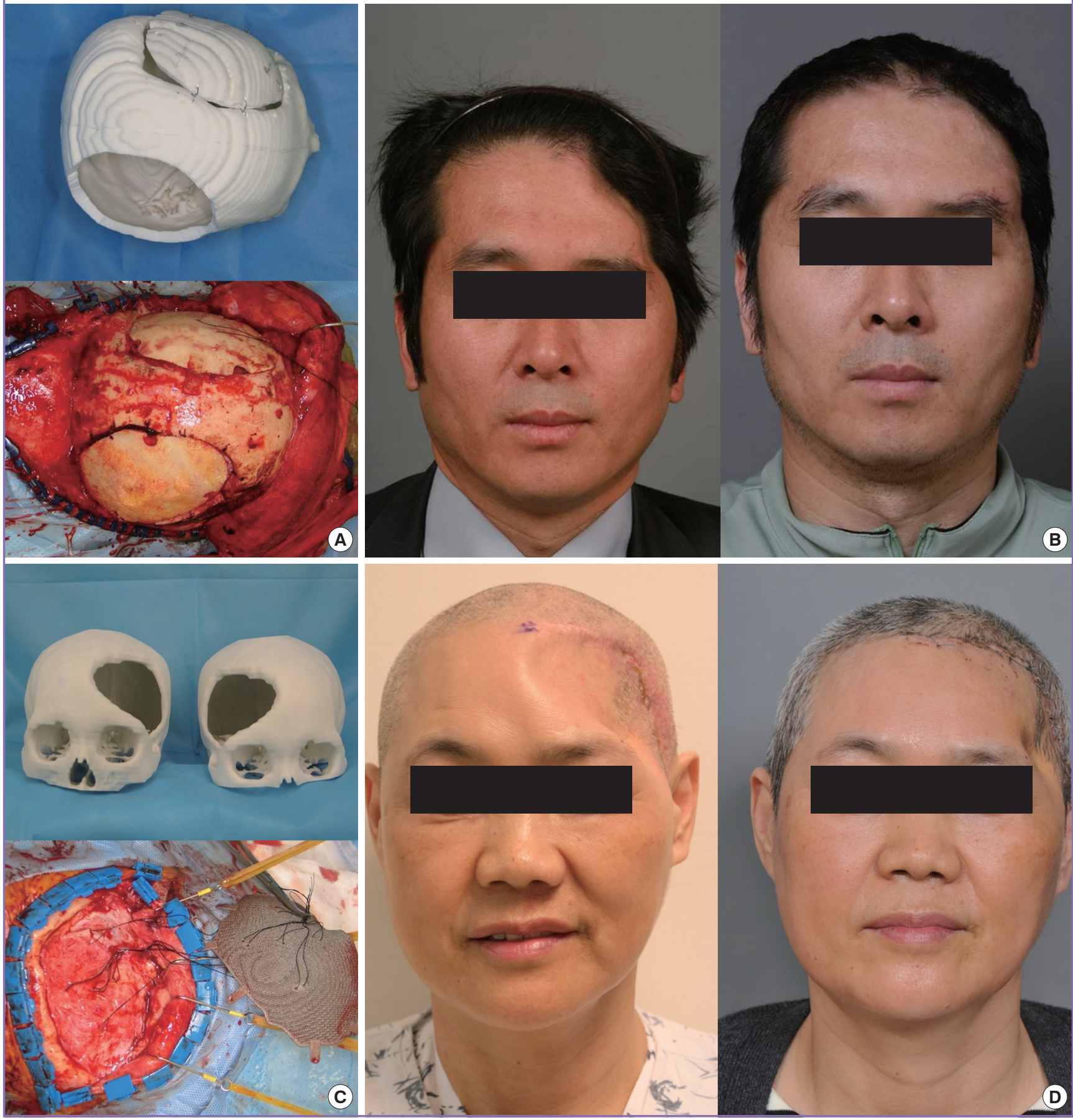


\section{Clinical applications for 3D printing-based tactile prototype models in craniofacial surgery}

Skull reconstruction

Calvarial bone reconstruction would be the most pioneering use of RP models. In 1994, Mankovich et al. [12] first applied 3D technology for skull reconstruction. Because autogenous bone grafting would be the ideal standard for skull reconstruction, donor bone should be harvested. The ideal curvature should be researched in advance because the bone is so rigid that bending is quite difficult and risky [19]. Experience has shown that tactile prototype models are very helpful for identifying the ideal donor site. For example, skull reconstruction can be done with a split calvarial bone grafting technique and the ideal donor area can be determined in advance to match the ideal recipient calvarial bone curvature [20]. Although many materials can be used for 3D printing, few materials can be permanently inserted into the human body. Thus far, titanium is the ideal realistic material for human body use. Therefore, once the recently developed 3D technology starts to provide 3D titanium-based implants, they could be used in the human body [21-23]. The technique currently appears to be quite successful and without any complications. The implants fit very well onto pre-existing defects such as calvarium or maxillary defects. However, because the resection and reconstruction should be done at the same time, the possible defect cannot be easily estimated in advance because the extent of the resection could vary according to the intraoperative findings. Therefore, for the successful adoption of 3D printed titanium implants, the preoperative simulation should be precise and an intraoperative resection guide should be provided based on the preoperative simulation. In addition, additional problems such as the interaction of bone or muscle with titanium should be considered (Fig. 2).

Cranioplasty for the correction of syndromic craniosynostosis Similar to skull reconstruction with bone grafting, extensive bone grafting is needed to correct craniosynostosis. Current $3 \mathrm{D}$ printing technology can provide an osteotomy guide that is very useful for the reconstruction process. Moreover, surgeons can simulate the surgery in advance using the 3D printed tactile model [24]. 3D printed prototype models are a particularly effective tool for simulating LeFort I, II, or III midface osteotomy, which require delicate blind osteotomy [25] (Fig. 3).

\section{Facial bone fractures}

Although many facial bone fractures can be managed with 3D printing technology, orbital wall fractures would be the ideal target for these methods $[1,7,9,26]$. The orbit has such a complex anatomy that ideal reconstruction is not particularly easy. Unless the orbital wall is repaired very precisely, postoperative enophthalmos or diplopia can occur. Nevertheless, limited surgical fields during surgery of orbital wall fractures often causes reconstruction in the wrong plane. These kinds of difficulties can be overcome using 3D printed titanium mesh implants or prevent the malpositioning of implant material. Medpore or titanium mesh based on the RP model that are manufactured from the mirroring technique of CAD-CAM [23]. The contralateral orbit can be a reference. Using the contralateral orbital anatomy, ideal ipsilateral orbital structures can be simulated on computer software and can be manufactured using $3 \mathrm{D}$ printing technology. Based on this RP model, orbital wall reconstruction can be successfully performed (Fig. 4).

\section{Fig. 3. Syndromic craniosynostosis correction using 3D printing technology}

Syndromic craniosynostosis correction using three-dimensional (3D) printing technology. (A) A cranial osteotomy can be performed based on a $3 \mathrm{D}$ printed rapid prototype model that can provide the precise locations of the osteotomy and indicate sites of potential problems. (B) Based on the rapid prototype model, the craniotomy can be facilliated avoiding dural injury.
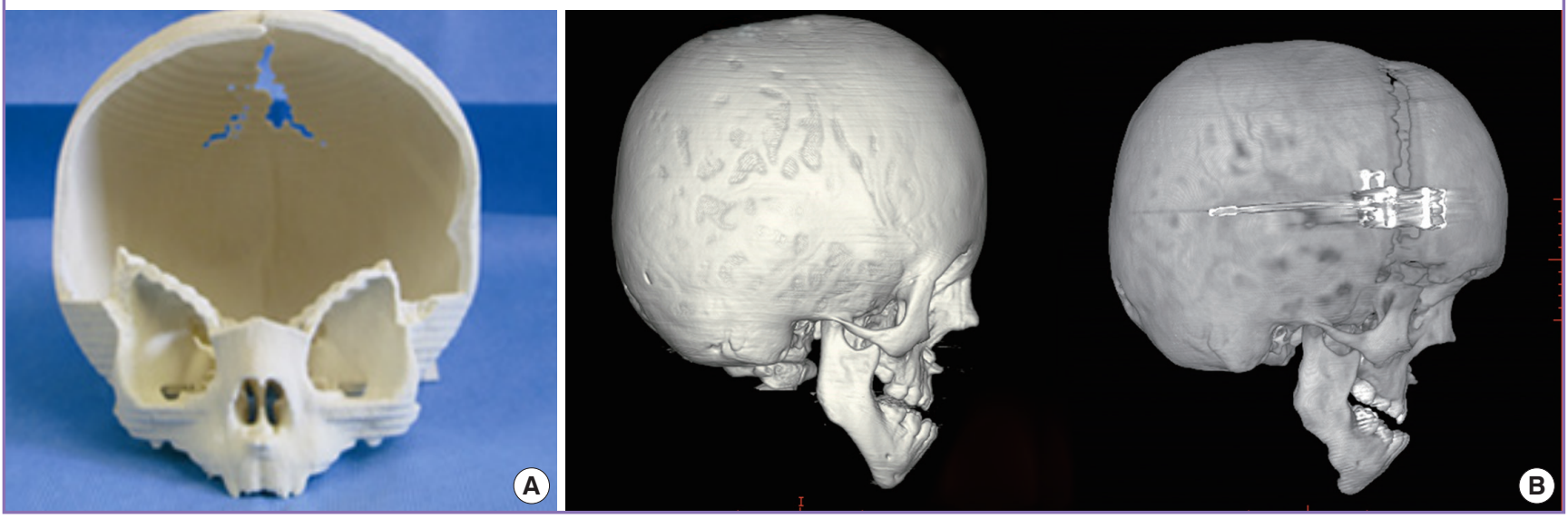
Orthognathic surgery

Numerous articles have been already published on the use of CAD-CAM technology for orthognathic surgery $[27,28]$. More- over, 3D printing technology provides an additional osteotomy guide and occlusal splint. However, the $3 \mathrm{D}$ printing model generally shows some errors in terms of accuracy, which is problem-

\section{Fig. 4. Orbital wall reconstruction based on a 3D printing}

Orbital wall reconstruction based on a three-dimensional (3D) printed tactile prototype model that was made by mirroring, computer-aided manufacturing (CAD-CAM) technology. (A) Mirrored image-based 3D printed tactile model. (B) The pre and postoperative computed tomography scans in orbital wall fracture.
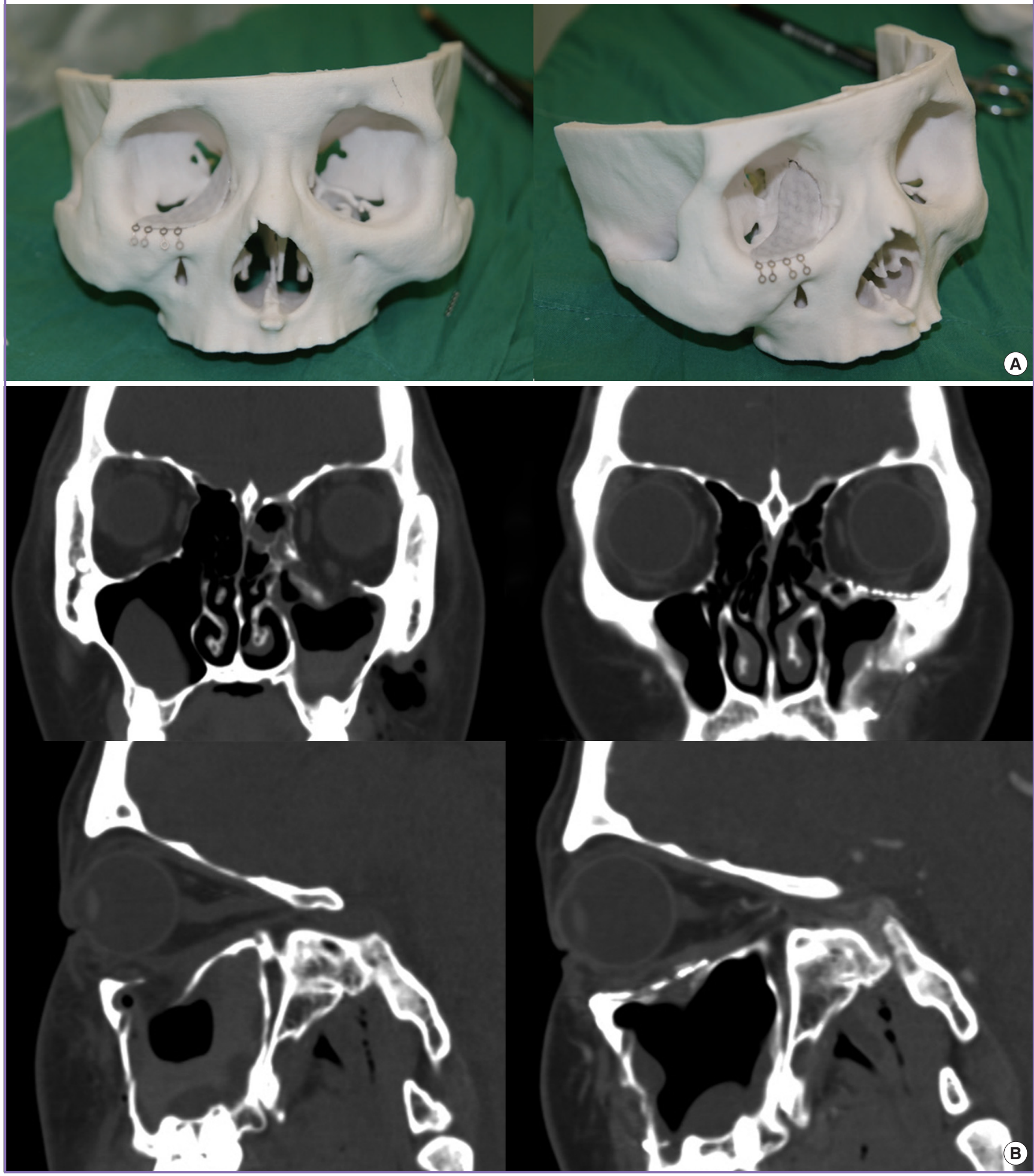


\section{Fig. 5. Orthognathic surgery can benefit from 3D printing}

Orthognathic surgery can benefit from three-dimensional (3D) printing technology. (A) Various anatomic landmarks on the 3D printed model can be found preoperatively, which enable the surgeon to perform the satisfactory surgical process. In addition, this is very useful for the consultation with patients and the presurgical simulational surgery. (B) In case of severe asymmetry, the rapid prototype model can provide 3 dimensional images for the surgeons.
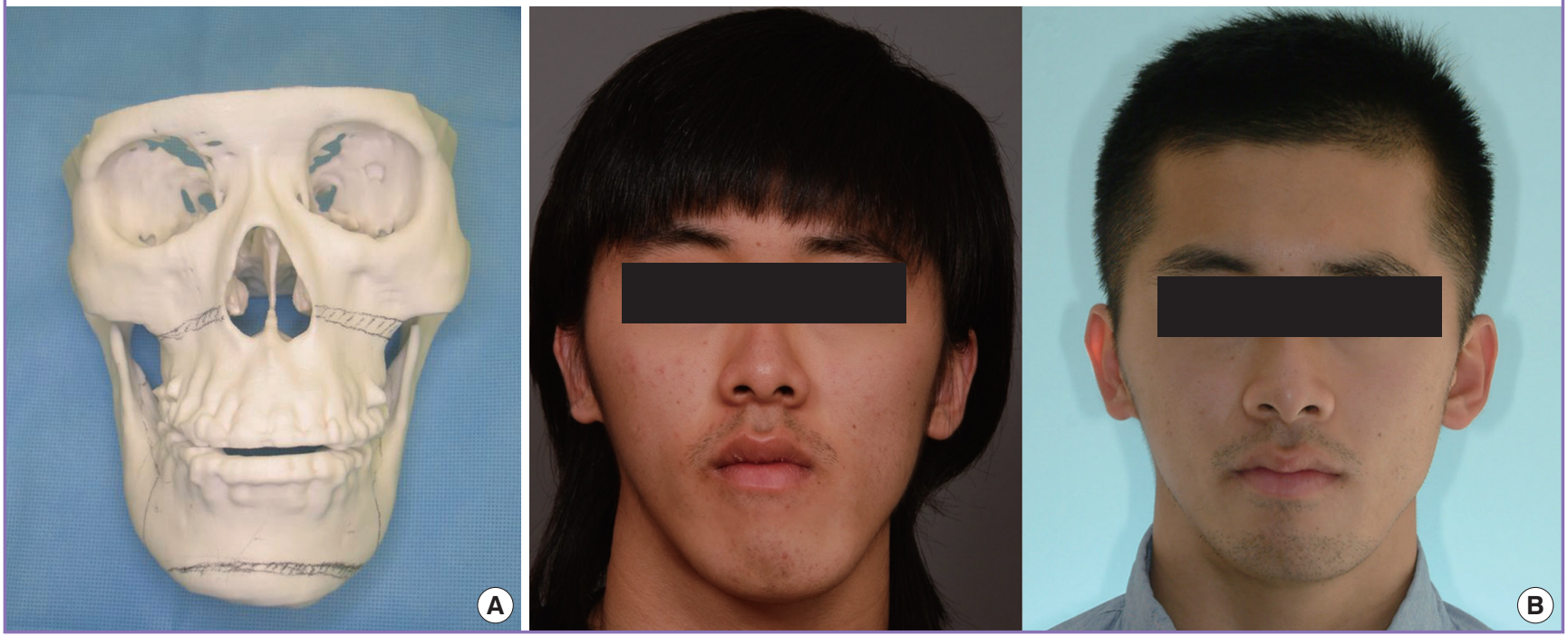

Fig. 6. Maxillary reconstruction with a 3D printing

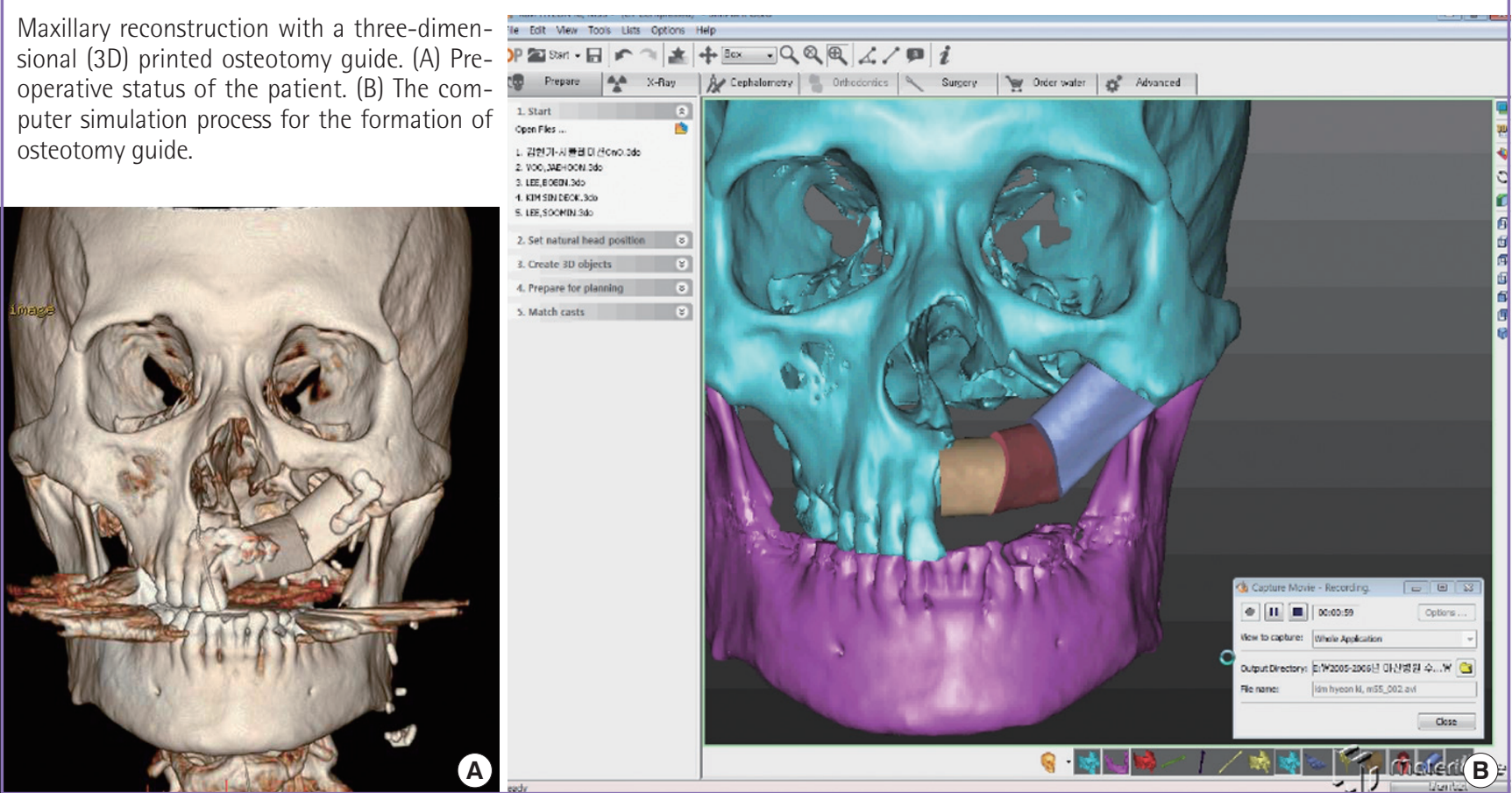

atic for ideal dental occlusion. Therefore, especially for dentition, scanning devices should be used to obtain ideal dental occlusal splints for orthognathic surgery (Fig. 5).

\section{Maxillary reconstruction}

Many articles using CAD-CAM technology have also been pub- lished. Recent advances in 3D printing technology now enable fibular osteotomy and fixation guides to be provided, which enable the dental implant to be inserted in the ideal position $[22,29]$ (Fig. 6). 


\section{Fig. 7. Mandibular reconstruction with a 3D printing}

Mandibular reconstruction with a three-dimensional (3D) printed osteotomy guide. (A) Computer simulation process can be realized into the real size 3D printed model. (B) Based on the 3D printing model from the computer simulation, ideal curvature of the mandible can be reconstructed for the dental implantation in the future.
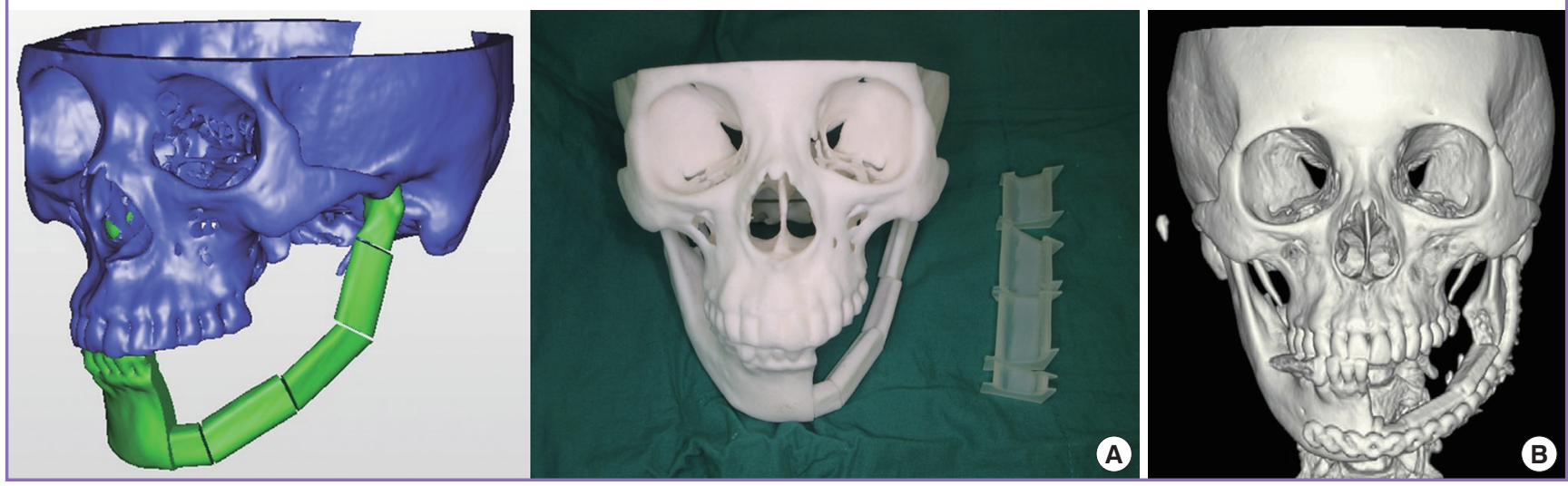

\section{Mandibular reconstruction}

Mandibular reconstruction is mostly being performed using fibular osteocutaneous free flaps. Although the curvature of the original mandible can be reconstructed using the conventional method, 3D CAD-CAM technology can provide a more precise reconstruction modality that includes fibular osteotomy and fixation guides [30,31]. In addition, 3D printed titanium fixation plates were recently tried and have been shown to be very useful for the ideal reconstruction of the mandible. In the near future, 3D printed titanium implants that can be inserted into the human body as a whole might be possible (Fig. 7).

\section{DISCUSSION}

Although 3D printing technology is evolving, its clinical applications are actually evolving more rapidly. The affordability and convenience of this technology have spurred its adoption in a variety of medical fields. Although there are still insufficient scientific data, 3D printing technology is innovating medicine. For example, pediatric cardiac surgeons are using $3 \mathrm{D}$ printing-based tactile models for analysing and visualizing complex congenital heart diseases. Urologic surgeons are simulating surgery of complex renal cell carcinoma in advance using $3 \mathrm{D}$ printed tactile prototype models that include the vessels and parenchyma of the kidney. Neurosurgeons are using similar approaches for neurosurgery of brain tumours. These kinds of efforts afford the various types of surgeons involved huge benefits by aiding in the advance analysis of the patient's specific status. In addition, these kinds of tactile models can be very helpful for preoperative consultations with patients and as a real intraoperative 1:1 scale reference $[3,8]$.

Meanwhile, tissue engineers are also seeing the advent of a new era. The tissue engineering triad comprises cell, scaffold, and growth factor. Recently, 3D technology has become sufficiently evolved to enable bio-cell printing. Although many obstacles need to be overcome, 3D bio-cell printing is providing bioengineers with a new modality that might be superior to the conventional cell culture systems. In reality however, 3D cell culture on scaffolds has a big limitation in technology and $3 \mathrm{D}$ bio-cell printing technology may overcome this obstacle sooner or later. In addition, the scaffold can be constructed using 3D technology. Therefore, in the near future, the simultaneous formation of the bio-cell and scaffolds might be possible based on $3 \mathrm{D}$ bio-cell printing technology. Of course, many obstacles still remain [1,32-36].

$3 \mathrm{D}$ printing techniques have been most actively used in craniofacial surgery, as reviewed here and based on extensive experience with $3 \mathrm{D}$ printing in craniofacial reconstruction. However, some obstacles need to be overcome.

First, the computer software used for craniofacial reconstruction should be much more specifically designed. The preoperative design of surgery is not especially easy however. Because the segmentation process in computer simulations is time consuming, it needs to be more automated. If the various software programs were more suitable and specific for craniofacial reconstruction, the $3 \mathrm{D}$ printing technique could be more actively used.

Second, a connection between the preoperative simulations and the real surgery environment should be made. Surgical wafers, such as intermediate and final dental splints, would be an example in orthognathic surgery. In addition, a navigational system could act as a surgical guide to connect the preoperative simulation and the actual surgery. In order to apply the $3 \mathrm{D}$ printed titanium implant, the surgical cut or ostectomy should be matched precisely with the preoperative planning. Because the $3 \mathrm{D}$ printed implant is so solid that it is not easy to cut or bend, planning 
and surgery should be identical and efforts should be made to ensure that the preoperative planning and intraoperative defect are in agreement. Therefore, a surgical osteotomy guide should be made.

A third issue is accuracy. Although CT scans are made in very thin slices, the imaging modality can only provide the accumulation of the multiple slices. Error can inevitably occur between the slices. In particular, the orbital wall is too thin to be reconstructed by only a $3 \mathrm{D}$ printing technique and a $3 \mathrm{D}$ printed orbit model represents the orbit as vacant fields.

Finally, the artifacts associated with metal can discourage the use of 3D printing models. For example, dental models cannot be recreated with CT scanning because of accuracy issues. Dental occlusion requires such delicate precision that the $3 \mathrm{D}$ rendering of CT data cannot provide a sufficiently high resolution. Hence, dental scanners are currently being used and techniques that merge $\mathrm{CT}$ and dental scans are common.

Despite these obstacles, 3D printing technology could be a new medical modality. Although this basic technology was initially developed over 20 years ago, further technological advances could enable medical doctors to realize patient-specific individualized medicine in the near future.

\section{CONCLUSIONS}

3D printing technology enables more effective patient consultations, increases diagnostic quality, improves surgical planning, acts as an orientation aid during surgery, and provides a template for surgical resection. In addition, as bio-cell printing technology further evolves, tissues or organs might one day be made using 3D printing methods. 3D printing technology thus has the potential to be very beneficial to patients and doctors in terms of patient-specific individualized medicine.

\section{REFERENCES}

1. Fullerton JN, Frodsham GC, Day RM. 3D printing for the many, not the few. Nat Biotechnol 2014;32:1086-7.

2. Hoy MB. 3D printing: making things at the library. Med Ref Serv Q2013;32:94-9.

3. Winder J, Bibb R. Medical rapid prototyping technologies: state of the art and current limitations for application in oral and maxillofacial surgery. J Oral Maxillofac Surg 2005;63: 1006-15.

4. Lee $\mathrm{M}, \mathrm{Wu} \mathrm{BM}$. Recent advances in $3 \mathrm{D}$ printing of tissue engineering scaffolds. Methods Mol Biol 2012;868:257-67.

5. Gross BC, Erkal JL, Lockwood SY, et al. Evaluation of 3D printing and its potential impact on biotechnology and the chemical sciences. Anal Chem 2014;86:3240-53.

6. Romano PE. 3D Printing Surgical Models of Organs; or Lunch; Phone Screens; How To Do It (3D) Yourself; Computers that Track Your Eyes and Take Eye Commands. Binocul Vis Strabolog Q Simms Romano 2013;28:121-8.

7. Michalski MH, Ross JS. The shape of things to come: 3D printing in medicine. JAMA 2014;312:2213-4.

8. Ventola CL. Medical applications for 3D Printing: current and projected uses. P T 2014;39:704-11.

9. Olszewski R, Tranduy K, Reychler H. Innovative procedure for computer-assisted genioplasty: three-dimensional cephalometry, rapid-prototyping model and surgical splint. Int J Oral Maxillofac Surg 2010;39:721-4.

10. Winder J, McRitchie I, McKnight W, et al. Virtual surgical planning and $\mathrm{CAD} / \mathrm{CAM}$ in the treatment of cranial defects. Stud Health Technol Inform 2005;111:599-601.

11. Olszewski R, Reychler H. Clinical applications of rapid prototyping models in cranio-maxillofacial surgery. In: Hoque $\mathrm{M}$, editor. Advanced applications of rapid prototyping technology in moderm engineering. Belgium: InTech; 2011.p.173206.

12. Mankovich NJ, Samson D, Pratt W, et al. Surgical planning using three-dimensional imaging and computer modeling. Otolaryngol Clin North Am 1994;27:875-89.

13. Choi JY, Choi JH, Kim NK, et al. Analysis of errors in medical rapid prototyping models. Int J Oral Maxillofac Surg 2002; 31:23-32.

14. Chang PS, Parker TH, Patrick CW Jr, et al. The accuracy of stereolithography in planning craniofacial bone replacement. J Craniofac Surg 2003;14:164-70.

15. Schicho K, Figl M, Seemann R, et al. Accuracy of treatment planning based on stereolithography in computer assisted surgery. Med Phys 2006;33:3408-17.

16. Ibrahim D, Broilo TL, Heitz C, et al. Dimensional error of selective laser sintering, three-dimensional printing and PolyJet models in the reproduction of mandibular anatomy.J Craniomaxillofac Surg 2009;37:167-73.

17. Silva DN, Gerhardt de Oliveira M, Meurer E, et al. Dimensional error in selective laser sintering and 3D-printing of models for craniomaxillary anatomy reconstruction. J Craniomaxillofac Surg 2008;36:443-9.

18. Ohtani T, Kusumoto N, Wakabayashi K, et al. Application of haptic device to implant dentistry: accuracy verification of drilling into a pig bone. Dent Mater J 2009;28:75-81.

19. Chang TJ, Choi JW, Ra YS, et al. Changes in graft thickness after skull defect reconstruction with autogenous split calvarial bone graft. J Craniofac Surg 2014;25:1241-4.

20. Lee HJ, Choi JW, Chung IW. Secondary skull reconstruc- 
tion with autogenous split calvarial bone grafts versus nonautogenous materials. J Craniofac Surg 2014;25:1337-40.

21. Chen ST, Chang CJ, Su WC, et al. 3-D titanium mesh reconstruction of defective skull after frontal craniectomy in traumatic brain injury. Injury 2015;46:80-5.

22. Biron VL, Gross M, Broad R, et al. Radial forearm free flap with titanium mesh sandwich reconstruction in complex anterior skull base defects. J Craniofac Surg 2012;23:1763-5.

23. Beliakin SA, Khyshov VB, Khyshov MB, et al. Reconstruction of posttraumatic skull and facial bones injuries with the use of perforated titanium plates and meshes. Voen Med $\mathrm{Zh}$ 2012;333:12-7.

24. Choi JW, Koh KS, Hong JP, et al. One-piece frontoorbital advancement with distraction but without a supraorbital bar for coronal craniosynostosis. J Plast Reconstr Aesthet Surg 2009;62:1166-73.

25. Choi JW, Ra YS, Hong SH, et al. Use of distraction osteogenesis to change endocranial morphology in unilateral coronal craniosynostosis patients. Plast Reconstr Surg 2010;126: 995-1004.

26. Malhotra K, Sharma A, Giraddi G, et al. Versatility of titanium $3 \mathrm{D}$ plate in comparison with conventional titanium miniplate fixation for the management of mandibular fracture. J Maxillofac Oral Surg 2012;11:284-90.

27. Zinser MJ, Mischkowski RA, Sailer HF, et al. Computer-assisted orthognathic surgery: feasibility study using multiple CAD/CAM surgical splints. Oral Surg Oral Med Oral Pathol Oral Radiol 2012;113:673-87.
28. Okumura H, Chen LH, Yokoe Y, et al. CAD/CAM fabrication of occlusal splints for orthognathic surgery. J Clin Orthod 1999;33:231-5.

29. Lethaus B, Kessler P, Boeckman R, et al. Reconstruction of a maxillary defect with a fibula graft and titanium mesh using CAD/CAM techniques. Head Face Med 2010;6:16.

30. Xu X, Ping FY, Chen J, et al. Application of CAD/CAM techniques in mandible large-scale defect and reconstruction with vascularized fibular bone graft. Zhejiang Da Xue Xue Bao Yi Xue Ban 2007;36:498-502.

31. Zhang T, Zhang Y, Li YS, et al. Application of CTA and CAD \ CAM techniques in mandible reconstruction with free fibula flap. Zhonghua Zheng Xing Wai Ke Za Zhi 2006;22:325-7.

32. Dankowski R, Baszko A, Sutherland M, et al. 3D heart model printing for preparation of percutaneous structural interventions: description of the technology and case report. Kardiol Pol 2014;72:546-51.

33. Rengier F, Mehndiratta A, von Tengg-Kobligk H, et al. 3D printing based on imaging data: review of medical applications. Int J Comput Assist Radiol Surg 2010;5:335-41.

34. McGowan J. 3D printing technology speeds development. Health Estate 2013;67:100-2.

35. Lee $\mathrm{H}$, Fang NX. Micro 3D printing using a digital projector and its application in the study of soft materials mechanics. J Vis Exp 2012:e4457.

36. Mironov V, Boland T, Trusk T, et al. Organ printing: computer-aided jet-based 3D tissue engineering. Trends Biotechnol 2003;21:157-61. 